\title{
The Role of Wild-Type p53 in Cisplatin-Induced Chk2 Phosphorylation and the Inhibition of Platinum Resistance with a Chk2 Inhibitor
}

\author{
Xiaobing Liang, ${ }^{1}$ Yi Guo, ${ }^{1}$ William Douglas Figg, ${ }^{2}$ Antonio Tito Fojo, ${ }^{3}$ \\ Michael D. Mueller, ${ }^{1}$ and Jing Jie Yu${ }^{1}$ \\ ${ }^{1}$ Department of Biochemistry, School of Medicine, Department of Basic Pharmaceutical Sciences, School of Pharmacy, \\ and Mary Babb Randolph Cancer Center, Robert C. Byrd Health Sciences Center, West Virginia University, Morgantown, \\ WV 26506, USA \\ ${ }^{2}$ Molecular Pharmacology Section, Medical Oncology Branch and Affiliates, National Cancer Institute, \\ National Institutes of Health, Bethesda, MD 20892, USA \\ ${ }^{3}$ Experimental Therapeutics Section, Medical Oncology Branch and Affiliates, National Cancer Institute, National Institutes of Health, \\ Bethesda, MD 20892, USA
}

Correspondence should be addressed to Jing Jie Yu, jyu@hsc.wvu.edu

Received 26 August 2010; Accepted 25 October 2010

Academic Editor: Athanassios Tsakris

Copyright (c) 2011 Xiaobing Liang et al. This is an open access article distributed under the Creative Commons Attribution License, which permits unrestricted use, distribution, and reproduction in any medium, provided the original work is properly cited.

\begin{abstract}
The major obstacle in platinum chemotherapy is the repair of platinum-damaged DNA that results in increased resistance, reduced apoptosis, and finally treatment failure. Our research goal is to determine and block the mechanisms of platinum resistance. Our recent studies demonstrate that several kinases in the DNA-repair pathway are activated after cells are exposed to cisplatin. These include ATM, p53, and Chk2. The increased Chk2 phosphorylation is modulated by p53 in a wild-type p53 model. Overexpression of p53 by cDNA transfection in wt-p53 (but not p53 deficient) cells doubled the amount of Chk2 phosphorylation 48 hours after cisplatin treatment. p53 knockdown by specific siRNA greatly reduced Chk2 phosphorylation. We conclude that wild-type p53, in response to cisplatin stimulation, plays a role in the upstream regulation of Chk2 phosphorylation at Thr-68. Cells without normal p53 function survive via an alternative pathway in response to the exogenous influence of cisplatin. We strongly suggest that it is very important to include the p53 mutational status in any p53 involved studies due to the functional differentiation of wt p53 and p53 mutant. Inhibition of Chk2 pathway with a Chk2 inhibitor (C3742) increased cisplatin efficacy, especially those with defective p53. Our findings suggest that inhibition of platinum resistance can be achieved with a small-molecule inhibitor of Chk2, thus improving the therapeutic indices for platinum chemotherapy.
\end{abstract}

\section{Introduction}

DNA targeting agents for cancer treatment are among the most common treatments to fight cancer. However, side effects and drug resistance of chemotherapy are a major clinical problem, seriously affecting both the life quality of patients and the outcome of treatment. Studies show that cells treated with genotoxic agents swiftly respond by activating DNA-damage checkpoint response. This prompts the repair of DNA lesions while transiently slowing down replication, or it elicits an apoptotic program in case of massive or irreparable lesions to the DNA. Two primary pathways are initiated in response to DNA damage. One is mediated by the ATM-Chk2 axis and the other, via the ATRChk1 axis. The ATM/Chk2 pathway responds primarily to DNA double-strand breaks, whereas the ATR-Chk1 pathway mainly responds to replication-associated DNA lesions $[1,2]$.

The transcription factor p53, a DNA-binding protein containing DNA-binding, oligomerization, and transcriptional activation domains [3-6], plays a key role in the DNA damage response to genotoxic stress by binding directly to the promoters of target genes and altering the rate at which they are transcribed $[7,8]$. Under normal conditions wildtype p53 is maintained at a low level due to the extremely 
short half-life of the polypeptide, and in a largely inactive state that is inefficient for its function [7-10]. In response to genotoxic stress, wt p53 proteins form a tetramer that binds DNA for exerting its transactivate function $[6,11]$. This will lead to the activation of numerous genes that cause growth arrest and apoptosis [7-11].

Checkpoint kinase $2(\mathrm{Chk} 2)$, a serine/threonine kinase and encoded protein, contains a forkhead-associated protein interaction domain. It lies at the heart of the DNA damage/repair pathway and is responsible for the maintenance of mammalian genomic integrity. Following exposure to ionizing radiation, Chk2 is rapidly activated by ATM and DNA-PK (DNA-dependent protein kinase) via phosphorylation at $\mathrm{Thr}^{68}$ of $\mathrm{Chk} 2$, causing homodimerization and subsequent trans-activating autophosphorylations at $\mathrm{Thr}^{383}$ and $\mathrm{Thr}^{387}$ and cis-phosphorylation at $\operatorname{Ser}^{516}[1,2,12]$. Chk2 activation occurs in an ATM-independent manner in response to UV radiation or stalled DNA replication [13]. In turn, activated Chk2 phosphorylates downstream substrates, including Cdc25A on serine ${ }^{123}$ and Cdc25C on Ser ${ }^{215}$, and inhibits Cdc25C phosphatase, preventing entry into mitosis, leading to cell cycle arrest in G1 phase. This protein also interacts with and phosphorylates BRCA1, allowing it to restore survival after DNA damage $[2,12,14,15]$.

Recent studies suggest that Chk2 inhibition in combination with genotoxic agents might have therapeutic value. Inhibition of Chk2 expression reduces DNA damageinduced cell cycle checkpoints and enhances apoptosis in p53-defective HEK-293 cells $[12,16]$. Chk2 inhibition also increases the level of mitotic catastrophe and sensitizes proliferating cells to doxorubicin-induced apoptosis [17]. Molecular or genetic targeting of Chk2 prevents the release of survivin from mitochondria and enhances DNA damageinduced tumor cell apoptosis, thus inhibiting in vivo growth of resistant tumors, providing a rational approach for treating these tumors [18]. In addition to augmenting the effect of cytotoxic drugs, Chk2 inhibitors may elicit radioor chemoprotection of normal tissue via abrogation of p53dependent apoptosis [19].

This investigation focused on the cisplatin-induced activation and regulation of Chk2 and further defines the relationship between two central mediators, p53 and Chk2, of the DNA damage/repair signaling pathway. Our results suggest that, in specific conditions, Chk2 activation at $\mathrm{Thr}^{68}$ phosphorylation is regulated by $\mathrm{p} 53$ in response to cisplatin treatment in wt p53-contain cells, but not in p53-deficient cells, of human ovarian cancer. Using a Chk2 inhibitor to block this cellular pathway greatly enhanced the efficacy of cisplatin in cancer chemotherapy.

\section{Materials and Methods}

2.1. Cell Culture and Drug Treatment. Human ovarian cancer cell lines A2780, Caov-3, and SKOV-3 were used in the current investigation. Cells were cultured in monolayer using RPMI 1640 media supplemented with 10\% (v/v) fetal calf serum, $2 \mathrm{mM}$ L-glutamine, 0.2 units $/ \mathrm{mL}$ human insulin, 50 units $/ \mathrm{mL}$ penicillin, and $50 \mathrm{mg} / \mathrm{mL}$ streptomycin
(Life Technologies, Inc., Gaithersburg, MD, USA). Cells were grown in logarithmic growth at $37^{\circ} \mathrm{C}$ in a humidified atmosphere consisting of $5 \% \mathrm{CO}_{2}, 95 \%$ air. Cells were routinely recovered from frozen stocks and allowed to grow until $80 \%$ confluence. For all experiments, cells were plated the day before exposure to treatment.

Cisplatin (CDDP) (Sigma-Aldrich, St. Louis, MO) was prepared fresh daily by first dissolving it in phosphatebuffered saline (PBS), without $\mathrm{Ca}^{++}$or $\mathrm{Mg}^{++}$, at a concentration of $1 \mathrm{mg}$ per $\mathrm{ml}$, and then further diluting it into prewarmed media to achieve the $\mathrm{IC}_{50}$ doses of 5-day survival $(3 \mu \mathrm{M}$ for $\mathrm{A} 2780,10 \mu \mathrm{M}$ for Caov-3, and $75 \mu \mathrm{M}$ for SKOV-3 cells, resp.). For the time-course studies, cells were allowed to grow for $24 \mathrm{hr}$ and treated with cisplatin for $1 \mathrm{hr}$ at $\mathrm{IC}_{50}$ doses. At the end of $1 \mathrm{hr}$ exposure to cisplatin, cells were washed twice with PBS and then incubated with fresh drug-free media for specifically required period of time.

2.2. Protein Extraction and Western Blotting. Treated and untreated cells were extracted with whole cell lysis buffer (20 mM Tris- $\mathrm{HCl}$ (pH 7.5), $150 \mathrm{mM} \mathrm{NaCl}, 1 \mathrm{mM} \mathrm{Na} 2$ EDTA, $1 \mathrm{mM}$ EGTA, 1\% Triton, $2.5 \mathrm{mM}$ sodium pyrophosphate, $\beta$-glycerophosphate, $1 \mathrm{mM} \mathrm{Na} \mathrm{VO}_{4}, 1 \mathrm{mM}$ PMSF, $10 \mu \mathrm{g} / \mathrm{mL}$ leupeptin, $10 \mu \mathrm{g} / \mathrm{mL}$ aprotinin, and $5 \mu \mathrm{g} / \mathrm{mL}$ pepstatin) for $30 \mathrm{~min}$ before centrifugation (at $14,000 \mathrm{rpm}$ for $30 \mathrm{~min}$ at $\left.4^{\circ} \mathrm{C}\right)$. Supernatant was collected as whole cell lysate for Western blot analysis. Protein concentrations of extracts were determined by Bio-Rad Protein Assay kit (Bio-Rad, Hercules, CA) with bovine serum albumin as standard.

The whole cell lysates were separated on 12\% SDSpolyacrylamide gels and transferred to PVDF membrane (BIO-RAD) using standard electrophoresis and electroblotting procedures. Prestained molecular weight markers were purchased from Invitrogen (Carlsbad, CA). To reduce nonspecific binding, blots were preincubated for $1 \mathrm{hr}$ in a blocking buffer ( $5 \%$ nonfat dry milk, $1 \mathrm{X}$ TBS, and $0.1 \%$ Tween 20). Membranes were then incubated with primary antibodies overnight at $4^{\circ} \mathrm{C}$. The primary antibodies applied were anti-ATM, anti-p-p53 phosphoserine-15, anti-p-p53 phosphoserine-20, anti-p53, anti-Chk2 phosphothreonine68, anti-Chk2, anti-P48, and anti-P21. To demonstrate an equal loading of each sample, membranes were reprobed with $\beta$-actin using anti- $\beta$-actin antibody (Sigma). The signals of immunoreactive proteins were visualized using horseradish peroxidase-conjugated sheep antimouse or donkey antirabbit antibodies and enhanced by Chemiluminescence ECL detection system (Amersham, UK).

2.3. p53 Stable and Transient Transfection. A2780 and SKOV3 cells were transfected with the plasmid pC53-SN3 or pCMV-Neo-Bam (provided by Dr. Bert Vogelstein) for the expression of wild-type human p53. Twenty-four hours after transfection, cells were treated with cisplatin at $\mathrm{IC}_{50}$ doses for $1 \mathrm{hr}$ and then were continuously incubated with fresh media for indicated hours. Stable cell lines were established by selection of positive transfected clones that grew in media containing G418 prior to cisplatin treatment. Cell lysate 
was obtained by lysing the cells in buffer $(20 \mathrm{mM}$ HEPES (pH 7.0), $1 \mathrm{mM}$ DTT, $1 \mathrm{mM} \mathrm{MnCl}_{2}, 100 \mu \mathrm{g} / \mathrm{mL}$ BSA, and $50 \mu \mathrm{M}$ leupeptin) and performed for Western blot analysis.

2.4. siRNA-Mediated $p 53$ Silencing Assay. Small interfering RNAs (siRNAs) against p53 (SMARTpool p53) were purchased from Upstate Biotechnology (Lake Placid, NY). The siRNA Transfection Reagent Lipofectamine 2000 (Invitrogen) was used to transfect the siRNA into A2780 cells at a final concentration of $100 \mathrm{nM}$. A negative nonspecific siRNA was used as control. Twenty-four hours after transfection, cells were treated with cisplatin for $1 \mathrm{hr}$ at $\mathrm{IC}_{50}$ dose. At the end of $1 \mathrm{hr}$ exposure to cisplatin, cells were washed twice and then incubated with fresh drug-free media for indicated hours. The cells were harvested and protein level of $\mathrm{p}-\mathrm{Chk} 2$, Chk2, p-p53, p53, and P21 were determined by western blotting.

2.5. Chk2 Transient Transfection. A2780 cells were transfected with the plasmid pEF-BOS-HA, pEF-BOS-HA-Chk2, or pEF-BOS-HA-Chk2D347A (provided by Dr. Jann N. Sarkaria) for control or the expression of wild-type human Chk2 or the expression of Chk2 kinase-dead point mutant. Twenty-four hours after transfection, cells were treated with cisplatin, and then were measured their expression levels.

2.6. Cytotoxicity Assay. A2780 or Caov-3 cells (around 1000 per well) were seeded in 96-well plates for $24 \mathrm{hrs}$ and pretreated with different concentrations of Chk2 inhibitor II C3742 for $30 \mathrm{~min}$. Subsequently, cells were treated with cisplatin at $\mathrm{IC}_{50}$ dose for $1 \mathrm{hr}$ while continuously exposed to Chk2 inhibitor. After drug removal, cells were washed twice with PBS, and then incubated with fresh medium containing Chk2 inhibitor for 5 days. Cytotoxicity assay was performed following company's protocol of Cell Proliferation Kit I (Roche) and the complete solubilization of the purple formazan crystals was measured. Optical density was read at $600 \mathrm{~nm}$ to determine cell quantity.

\section{Results}

3.1. Cisplatin Induced Phosphorylation of p53 and Chk2 in A2780 Cells. Human ovarian cancer A2780 cells were analyzed for p53 mutations within exons 4 through 9 and classified to p53 wild-type cell line (data not shown). A2780 cells were exposed to Cisplatin at $3 \mu \mathrm{M}\left(\mathrm{IC}_{50}\right)$ for $1 \mathrm{~h}$, and Western blotting was performed to analyze the levels of selected proteins of DNA damage response pathway. We observed increased protein level of ATM, p53, P48, and P21 in a time-dependent manner (Figure 1). At $12 \mathrm{~h}$ after cisplatin treatment, the p53 level is highly accumulated, accompanied by 553 phosphorylation. After drug treatment, these cells demonstrated cisplatin-induced p53 phosphorylation at serine 15 and serine 20 and Chk 2 phosphorylation at Thr-68. Further, the greatest increase in p53 phosphorylation induced by cisplatin occurred 12 hours before the primary increase in Chk2 phosphorylation, hinting that p53 activation occurs before Chk2. In addition, we observed that

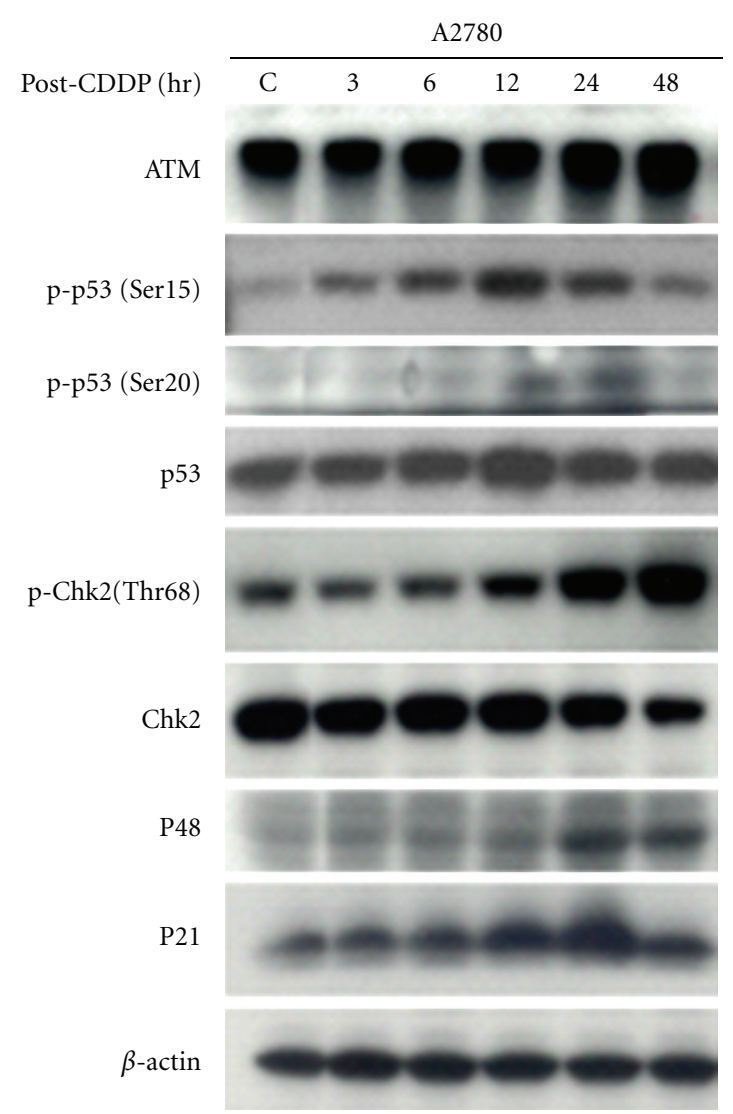

FIgURE 1: Cisplatin-induced DNA damage response in ovarian cancer A2780 cells. A2780 cells were treated with $3 \mu \mathrm{M}$ cisplatin (CDDP) for $1 \mathrm{hr}$. Cells were then washed to remove cisplatin and reincubated for indicated hours in a replacement of fresh drug-free media. Cells were harvested, and proteins were extracted, separated on SDS-PAGE gel, transferred onto PVDF membrane, and then probed with antibodies of anti-ATM, anti-p-p53 (Ser15 \& Ser20), anti-p53, anti-p-Chk2 (Thr68), anti-Chk2, anti-P48, anti-P21 or anti- $\beta$-Actin. $\beta$-Actin served as a control.

cisplatin-mediated activation of p53 resulted in activation of downstream proteins P48 and P21 (Figure 1).

3.2. Overexpression of p53 in wt p53-Contain Cells Increased Chk2 Phosphorylation. To determine the effect of p53 regulation on Chk2 activation, we transfected the p53 wildtype A2780 cells with plasmid pC53-SN3, which expresses the wild-type human p53, and pCMV-Neo-Bam, which is an empty vector of pC53-SN3. Twenty-four hours after transfection cells were treated with cisplatin at $3 \mu \mathrm{M}$. Figure 2 shows that Chk2 phosphorylation is increased after p53 cDNA transfection. The overexpression of both p53 protein and phosphorylated $\mathrm{p} 53$ in these cells by cisplatin induction and cDNA transfection at least doubled the amount of observed Chk2 phosphorylation 48 hours after cisplatin stimulation.

3.3. Transfection of p53 in p53-Null Cells Failed to Alter Chk2 Activation. We hypothesized that only the wt p53 


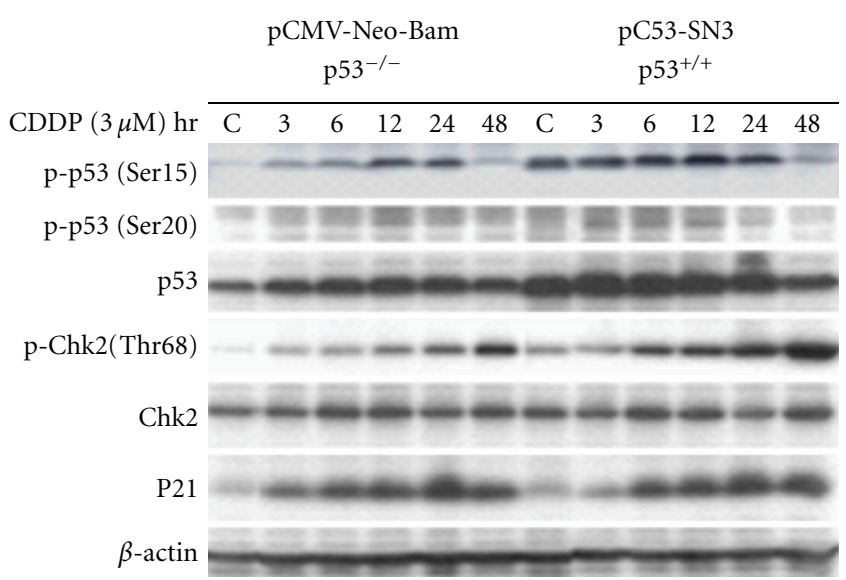

FIGURE 2: Overexpression of p53 by p53 transfection in A2780 cells. A2780 cells were transfected with the plasmid pC53-SN3 or pCMVNeo-Bam for overexpression of wild-type human p53. Twenty-four hours after transfection, cells were treated with cisplatin $(3 \mu \mathrm{M})$ for $1 \mathrm{hr}$ and then were continuously incubated with fresh media for indicated hours. Cell lysate was obtained by lysing the cells in lysate buffer and performed for Western blot analysis with antibodies of anti-p-p53(Ser15), anti-p-p53(Ser20), anti-p53, antip-Chk2(Thr68), anti-Chk2, anti-P21, or anti- $\beta$-Actin, respectively.

phenotype represents the p53 functional system. Therefore, we transfected the plasmid of pC53-SN3 and of pCMV-NeoBam vector into SKOV3, p53-null cells. These cells were also exposed to cisplatin after a 24-hour transfection. Western analysis demonstrated no effect of Chk2 phosphorylation by p53 stable transfection (Figure 3(a)) or p53 transient transfection (Figure 3(b)). The similar expression pattern of phosphorylated Chk2 induced by cisplatin alone was seen in the three sample groups of control, negative, and positive transfected cells. This indicates that cells without wt p53, to survive, may adapt via an alternative pathway in response to cisplatin treatment. In other words, cisplatin-induced Chk2 activation may be regulated following an alternative pathway in the p53-null cells.

3.4. Inhibition of p53 by Specific siRNA Inhibited Chk2 Phosphorylation. We performed p53 knockoff siRNA assay using small interfering RNA (siRNA) to silence the p53 then determine Chk2 expression in A2780 cells. The siRNA to human p53 contains 4 pooled SMART-selected siRNA duplexes with "UU" overhangs and a $5^{\prime}$ phosphate on the antisense strand (Upstate Biotechnology). As shown in Figure 4, in cells not treated with cisplatin, the siRNA to human p53 produced a decrease of phosphorylated Chk2, compared to the nonspecific siRNA-treated control. This decreased level may reflect a constitutive level of activated Chk2 68-phosphothreonine that is normally regulated by p53. Cells transfected by specific siRNA to p53 and treated with cisplatin resulted in a great reduction of p-Chk2 at Thr-68, suggesting that p53 modulates 68-threonine phosphorylation of Chk2.
3.5. Transfection of Chk2 in wt p53 Cells Did Not Alter p53 Level. Hypothetically, overexpression of Chk2 should not affect p53 level. To investigate this, we transfected the wt p53 A2780 cells with plasmid HA-Chk2, which expresses wild-type Chk2 (Figure 5(a)), and with HA-Chk2D347A, which is a kinase-dead Chk2 allele (Figure 5(b)). The empty vector was also transfected, respectively, as control. Cells were treated with cisplatin at $\mathrm{IC}_{50}$ dose $24 \mathrm{~h}$ after transfection. The results of Western blot analysis in these cells showed no effects of wild-type Chk2 (Figure 5(a)) or dead Chk2 (Figure 5(b)) on p53 protein or p53 phosphorylation (both Serine 15 and Serine 20). This suggests that Chk2 does not have a regulative effect on p53 in response to cisplatin treatment in ovarian cancer cells.

3.6. Application of Chk2 Inhibitor in Combination with Cisplatin Greatly Inhibited Cell Growth. Cytotoxicity Assay was performed to validate our hypothesis that application of Chk2 inhibitor potentiates cisplatin efficacy in ovarian cancer treatment. Figure 6 shows that selective Chk2 Inhibitor (C3742, Sigma) greatly increased the cell-killing effect of cisplatin in wt p53 A2780 cells (Figure 6(a)). In combination with Chk2 inhibitor at $75 \mu \mathrm{M}$, cisplatin produced about a 9fold inhibition on cell growth, compared to cisplatin alone. This effect appears to be more significant (nearly 38 -fold) in the mutant p53, Caov-3 cells (Figure 6(b)). We conclude that inhibition of Chk2 pathway with commercially available inhibitor will enhance the therapeutic indices of platinum compounds in the treatment of ovarian cancer, especially in those defective for p53 function.

\section{Discussion}

Disruption of the mechanisms that regulate checkpoint and apoptotic responses leads to genomic instability and the development of cancer [20]. It is in the best interest of the organism to prevent severely damaged cells from proliferating, either by halting them in a phase of cell cycle progression or by removing them entirely by apoptosis. The linear pathway of ATM-Chk2-p53 has been the dominant model and plays a central role in regulation of the cellular response to DNA damage, resulting in cell cycle arrest, DNA repair, or apoptosis depending on the cellular context and severity of DNA damage. In a well-studied example during doublestrand-DNA-break damage, the ATM phosphatidylinositol 3-kinase-like serine/threonine protein kinase is activated by autophosphorylation at the Ser ${ }^{1981}$ site [21]. Activated ATM then phosphorylates several downstream substrates, including Chk2 and p53 kinases. When the cell enters the damage-repair process, phosphorylated p53 arrests the cell cycle by inducing the expression of cell cycle inhibitors such as p21 [21]. On the other hand, Chk2 contributes to p53 stabilization in response to IR [22], suggesting that Chk2 may, in this circumstance, be an upstream regulator of p53 [23]. In addition, the abundance of Chk2 mRNA was shown to be inversely related to p53 status in human gastric cancer, indicating the possibility that Chk2 is a downstream target of the p53 [24-26]. Other evidence supports the model of 


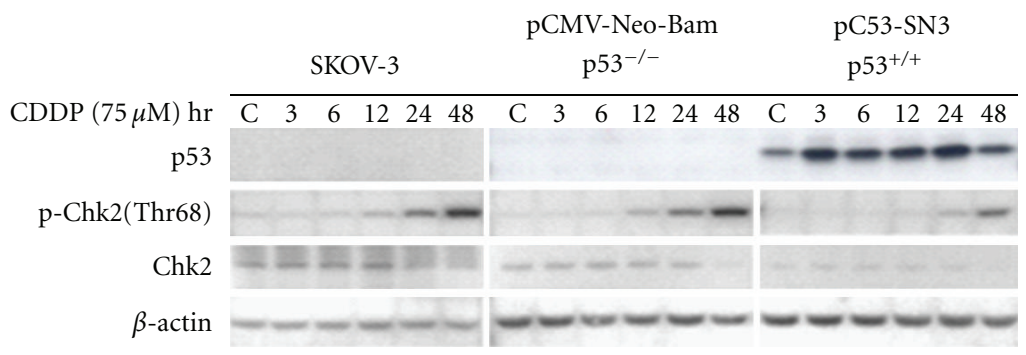

(a)

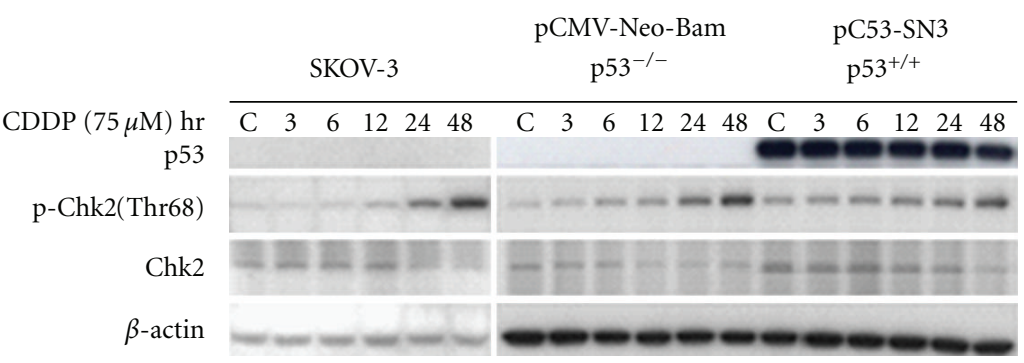

(b)

FIGURE 3: Transfection of p53 in p53-null SKOV-3 cells. Ovarian cancer SKOV-3 cells were transfected with the plasmid pC53-SN3 or pCMVNeo-Bam for $24 \mathrm{hr}$ : stable transfection (a) and transient transfection (b). Transfected cells were treated with cisplatin at $\mathrm{IC}_{50}$ dose ( $\left.75 \mu \mathrm{M}\right)$ for $1 \mathrm{hr}$. The drug was removed by washing the cells with PBS. Cells were then incubated in fresh drug-free media for the indicated hours $(3,6,12,24$, and 48$)$ up to harvest. Western blot analysis was performed with indicated antibodies. Nontransfected cells were treated with cisplatin alone as control group.

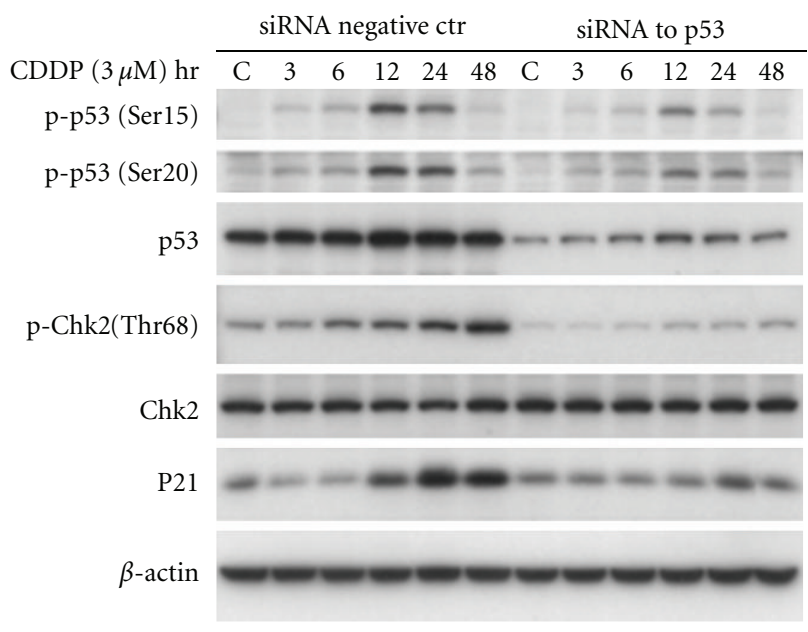

FIGURE 4: Inhibition of p53 by specific siRNA to p53 in A2780 cells. siRNA against p53 (SMARTpool p53) were transfected into A2780 cells using Lipofectamine 2000. A negative nonspecific siRNA was used as control. Twenty-four hours after transfection, cells were treated with cisplatin for $1 \mathrm{hr}$ at $3 \mu \mathrm{M}$ concentration. At the end of $1 \mathrm{hr}$ exposure to cisplatin, cells were washed and incubated with fresh media. The protein level of p-p53(Ser15), p-p53(Ser20), p53, p-Chk2(Thr68), Chk2, p21, and $\beta$-Actin were determined by Western blotting.

ATM-p53-Chk2, including: (a) Chk2 is not required for p53 responses in human cancer cells [27], (b) wild-type p53 suppresses mRNA and protein levels of Chk2 in human osteosarcoma Saos2 (p53 null) cells [28], (c) p53 mutations increase Chk2 expression in human gastric carcinoma [24], (d) Chk2 is dispensable for p53-mediated $\mathrm{G}_{1}$ arrest but is required for a latent p53-mediated apoptotic response [29], and (e) Chk2 expression is negatively regulated by functional p53, leading to a high level of expression in p53-deficient cancer cells $[20,30]$.

We observed that a cisplatin-induced increase in p53 phosphorylation preceded (by about 12 hours) the observed increase in Chk2 phosphorylation. Overexpression of p53 in studied cells by cDNA transfection doubled the amount of Chk 2 phosphorylation 48 hours after cisplatin treatment, whereas p53 knockdown by p53 specific siRNA greatly reduced Chk2 phosphorylation. In contrast, overexpression of Chk2 by transfection of wild-type Chk2 in these cells did not show effects on p53 protein or on phosphorylated p53 (Ser15 and Ser20). These observations further define the relationship between the two central mediators, p53 and Chk2. Our data suggest that in specific conditions Chk2 activation by $\mathrm{Thr}^{68}$ phosphorylation is regulated by p53 in response to cisplatin treatment in wt p53-contain cells, but not in p53-deficient cells, of human ovarian cancer. In addition, transfection of p53 cDNA into p53-null SKOV-3 cells failed to alter Chk2 activation, suggesting that the Chk2 role occurs alternatively and p53 independently in p53 defective cells. We conclude that wild-type p53, in response to cisplatin stimulation, plays a role in the upstream regulation of Chk2 phosphorylation at Thr-68. Cells without normal p53 function survive via an alternative pathway in response to the exogenous influence of cisplatin. We strongly suggest that it is very important to include the p53 mutational status in any p53 involved studies 


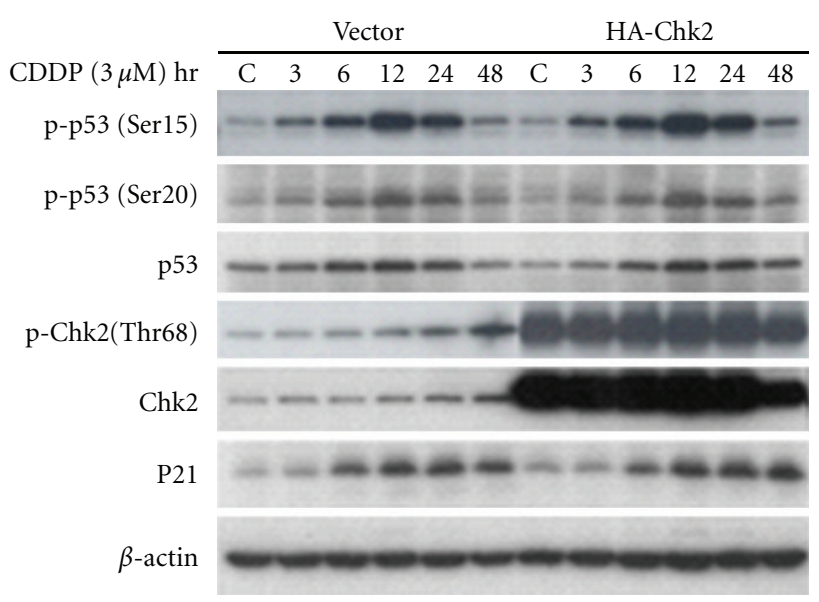

(a)

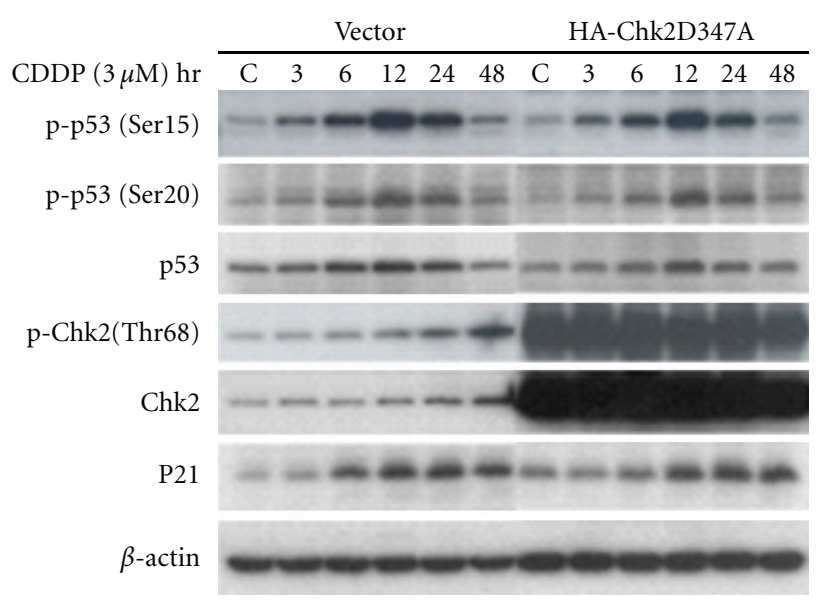

(b)

Figure 5: Overexpression of Chk2 by transfection of wild-type Chk2 or Chk2 dead allele in A2780 cells. A2780 cells were transfected with wild-type HA-Chk2 (a) or HA-Chk2 D347A (b) for overexpression of Chk2. Blank vectors were transfected as controls. Twenty-four hours after transfection, cells were treated with cisplatin $(3 \mu \mathrm{M})$ for $1 \mathrm{hr}$ and then were incubated with fresh media. Cell lysate was obtained and performed for Western blot analysis with antibodies of anti-p-p53(Ser15), anti-p-p53(Ser20), anti-p53, anti-p-Chk2(Thr68), anti-Chk2, anti-p21, or anti- $\beta$-Actin, respectively.

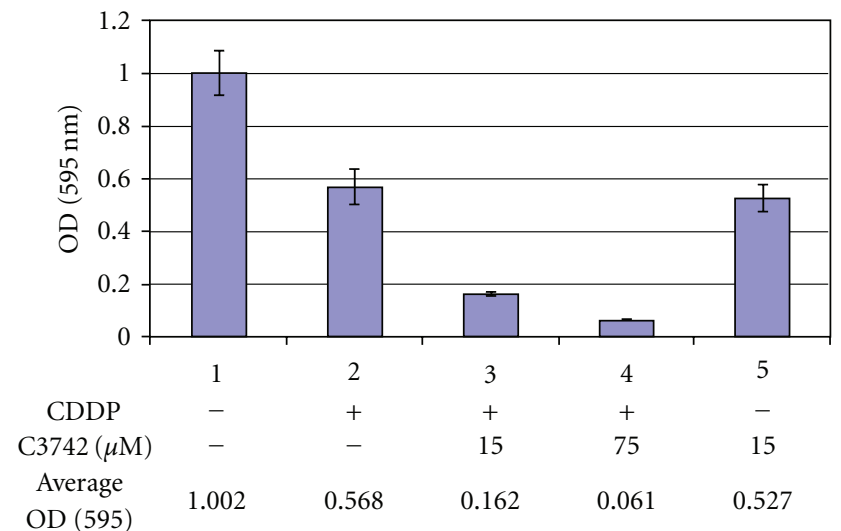

(a) Cell Model: p53 Wild Type

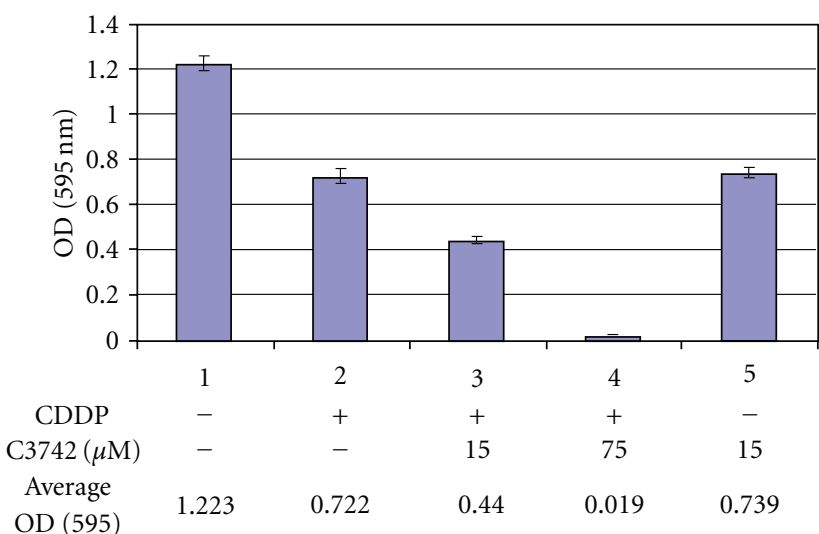

(b) Cell Model: p53 mutant

Figure 6: Application of Chk2 inhibitor in combination with cisplatin greatly inhibited cell growth. A2780 or Caov-3 cells (around 1000 per well) were seeded in 96-well plates for $24 \mathrm{hrs}$ and pretreated with different concentrations of Chk2 inhibitor II C3742 for 30 min. Subsequently, cells were treated with cisplatin at $\mathrm{IC}_{50}$ dose for $1 \mathrm{hr}$ while continuously exposed to Chk2 inhibitor. After drug removal, cells were washed twice with PBS, and then incubated with fresh medium containing Chk2 inhibitor for 5 days. Cytotoxicity assay was performed following company's protocol of Cell Proliferation Kit I (Roche) and the complete solubilization of the purple formazan crystals was measured. Optical density was read at $600 \mathrm{~nm}$ to determine cell quantity.

due to the functional differentiation of wt p53 and p53 mutant.

One of the approaches in current cancer treatment is to identify drugs acting on specific cancer-relevant targets. Specific sensitization of tumor cells to the action of genotoxins would reduce the efficacious doses of genotoxins to be used in patients, diminishing the detrimental side effects of the drugs on normal tissues $[31,32]$. The checkpoint kinase Chk2 is central to transmitting the DNA-damage signal. Parallel studies of the human Chk2 gene have highlighted its role as a candidate multiorgan tumor susceptibility gene rather than a tumor suppressor gene in the classical sense.
Chk2 can prevent tumor progression by averting genomic instability through DNA repair. If DNA-targeting agent is combined with a Chk2 inhibitor, DNA repair will be impaired and tumor cells unable to arrest the accumulation of irreparable damaged DNA and consequently undergo apoptosis [12]. Chk2 negatively regulate a poorly defined type of death occurring during mitosis or resulting from failed mitosis (mitotic catastrophe) by activating G2/M arrest and preventing entry into mitosis, meaning that inhibition of Chk2 may sensitize tumor cells to chemotherapy-induced apoptosis [17]. In addition, Chk2 has been shown to induce release from the mitochondria of the antiapoptotic protein 
survivin, which is thought to inhibit apoptosis in cancer cells and might confer radiation resistance in human cancer cells [18]. Chk2 elicits distinct cellular outcomes in the presence or absence of extrinsic DNA damage within a specific cellular context, in particular p53 status [12].

As stated earlier, the substrates of Chk2 kinase impact both cell cycle checkpoint and apoptosis. In a p53-deficient tumor, Chk2 primarily affects cell cycle checkpoint. Thus, Chk2 inhibition would abrogate DNA damage-induced cell cycle arrest and sensitize the tumor to chemotherapeutic agents. In normal tissues, Chk2 acts as a proapoptotic effector; therefore, a Chk2 inhibitor would protect normal tissues but sensitize the tumor to chemotherapeutic agents $[2,12,33]$. Given the known role of Chk2 in ovarian cancer pathogenesis and the findings from our studies and by other groups, our goal is to block the Chk2-regulated DNA repair pathway using a Chk2 inhibitor to enhance therapeutic index for current cancer chemotherapy.

\section{Acknowledgments}

The authors would like to thank Dr. Bert Vogelstein at The Johns Hopkins University School of Medicine for a permission to use the original construct pc53SN, which was provided by Dr. Curtis Harris in the Laboratory of Human Carcinogenesis, National Cancer Institute at Bethesda, MD, and thank Dr. Jann N. Sarkaria at Clinic of Mayo Foundation, Rochester, MN for providing the wild-type HA-hChk2 and mutated D347A plasmids for our investigation.

\section{References}

[1] J. Bartek and J. Lukas, "Chk1 and Chk2 kinases in checkpoint control and cancer," Cancer Cell, vol. 3, no. 5, pp. 421-429, 2003.

[2] Y. Pommier, J. N. Weinstein, M. I. Aladjem, and K. W. Kohn, "Chk2 molecular interaction map and rationale for Chk2 inhibitors," Clinical Cancer Research, vol. 12, no. 9, pp. 26572661, 2006.

[3] P. Lamb and L. Crawford, "Characterization of the human p53 gene," Molecular and Cellular Biology, vol. 6, no. 5, pp. 1379 $1385,1986$.

[4] G. Matlashewski, P. Lamb, D. Pim, J. Peacock, L. Crawford, and S. Benchimol, "Isolation and characterization of a human p53 cDNA clone: expression of the human p53 gene," EMBO Journal, vol. 3, no. 13, pp. 3257-3262, 1984.

[5] P. D. Jeffrey, S. Gorina, and N. P. Pavletich, "Crystal structure of the tetramerization domain of the p53 tumor suppressor at 1.7 angstroms," Science, vol. 267, no. 5203, pp. 1498-1502, 1995.

[6] Y. Cho, S. Gorina, P. D. Jeffrey, and N. P. Pavletich, "Crystal structure of a p53 tumor suppressor-DNA complex: understanding tumorigenic mutations," Science, vol. 265, no. 5170, pp. 346-355, 1994.

[7] N. D. Lakin and S. P. Jackson, "Regulation of p53 in response to DNA damage," Oncogene, vol. 18, no. 53, pp. 7644-7655, 1999.

[8] B. Vogelstein, D. Lane, and A. J. Levine, "Surfing the p53 network," Nature, vol. 408, no. 6810, pp. 307-310, 2000.

[9] G. Selivanova, "Mutant p53: the loaded gun," Current Opinion in Investigational Drugs, vol. 2, no. 8, pp. 1136-1141, 2001.
[10] T. Fojo, "p53 as a therapeutic target: unresolved issues on the road to cancer therapy targeting mutant p53," Drug Resistance Updates, vol. 5, no. 5, pp. 209-216, 2002.

[11] M. V. Blagosklonny, "p53: an ubiquitous target of anticancer drugs," International Journal of Cancer, vol. 98, no. 2, pp. 161166, 2002.

[12] L. Antoni, N. Sodha, I. Collins, and M. D. Garrett, "CHK2 kinase: cancer susceptibility and cancer therapy - two sides of the same coin?" Nature Reviews Cancer, vol. 7, no. 12, pp. 925936, 2007.

[13] A. Hirao, A. Cheung, G. Duncan et al., "Chk2 is a tumor suppressor that regulates apoptosis in both an ataxia telangiectasia mutated (ATM)-dependent and an ATM-independent manner," Molecular and Cellular Biology, vol. 22, no. 18, pp. 6521-6532, 2002.

[14] J. Falck, N. Mailand, R. G. Syljuåsen, J. Bartek, and J. Lukas, "The ATM-Chk2-Cdc25A checkpoint pathway guards against radioresistant DNA synthesis," Nature, vol. 410, no. 6830, pp. 842-847, 2001.

[15] J. Ahn and C. Prives, "Checkpoint kinase 2 (Chk2) monomers or dimers phosphorylate Cdc25C after DNA damage regardless of threonine 68 phosphorylation," Journal of Biological Chemistry, vol. 277, no. 50, pp. 48418-48426, 2002.

[16] Q. Yu, J. H. L. Rose, H. Zhang, and Y. Pommier, "Antisense inhibition of Chk2/hCds1 expression attenuates DNA damage-induced S and G2 checkpoints and enhances apoptotic activity in HEK-293 cells," FEBS Letters, vol. 505, no. 1, pp. 7-12, 2001.

[17] M. Castedo, J.-L. Perfettini, T. Roumier et al., "The cell cycle checkpoint kinase Chk2 is a negative regulator of mitotic catastrophe," Oncogene, vol. 23, no. 25, pp. 4353-4361, 2004.

[18] J. C. Ghosh, T. Dohi, C. M. Raskett, T. F. Kowalik, and D. C. Altieri, "Activated checkpoint kinase 2 provides a survival signal for tumor cells," Cancer Research, vol. 66, no. 24, pp. 11576-11579, 2006.

[19] A. G. Jobson, G. T. Lountos, P. L. Lorenzi et al., "Cellular inhibition of checkpoint kinase 2 (Chk2) and potentiation of camptothecins and radiation by the novel Chk2 inhibitor PV1019 [7-nitro-1H-indole-2-carboxylic acid \{4[1-(guanidinohydrazone)-ethyl]-phenyl - amide]," Journal of Pharmacology and Experimental Therapeutics, vol. 331, no. 3, pp. 816-826, 2009.

[20] H. Takai, K. Naka, Y. Okada et al., "Chk2-deficient mice exhibit radioresistance and defective p53-mediated transcription," EMBO Journal, vol. 21, no. 19, pp. 5195-5205, 2002.

[21] X. Yang, P. A. Wood, and W. J. M. Hrushesky, "Mammalian TIMELESS is required for ATM-dependent CHK2 activation and G2/M checkpoint control," Journal of Biological Chemistry, vol. 285, no. 5, pp. 3030-3034, 2010.

[22] N. H. Chehab, A. Malikzay, M. Appel, and T. D. Halazonetis, "Chk2/hCds1 functions as a DNA damage checkpoint in G1 by stabilizing p53," Genes and Development, vol. 14, no. 3, pp. 278-288, 2000.

[23] A. Hirao, Y. Y. Kong, S. Matsuoka et al., "DNA damageinduced activation of p53 by the checkpoint kinase Chk2," Science, vol. 287, no. 5459, pp. 1824-1827, 2000.

[24] H. Shigeishi, H. Yokozaki, N. Oue et al., "Increased expression of CHK2 in human gastric carcinomas harboring P53 mutations," International Journal of Cancer, vol. 99, no. 1, pp. 58-62, 2002.

[25] J. Ahn, M. Urist, and C. Prives, "Questioning the role of checkpoint kinase 2 in the p53 DNA damage response," Journal of Biological Chemistry, vol. 278, no. 23, pp. 2048020489, 2003 
[26] K. Tominaga, H. Morisaki, Y. Kaneko et al., "Role of human Cds1 (Chk2) kinase in DNA damage checkpoint and its regulation by p53," Journal of Biological Chemistry, vol. 274, no. 44, pp. 31463-31467, 1999.

[27] P. V. Jallepalli, C. Lengauer, B. Vogelsteint, and F. Bunz, "The Chk2 tumor suppressor is not required for p53 responses in human cancer cells," Journal of Biological Chemistry, vol. 278, no. 23, pp. 20475-20479, 2003.

[28] T. Matsui, Y. Katsuno, T. Inoue et al., "Negative regulation of Chk2 expression by p53 is dependent on the CCAAT-binding transcription factor NF-Y," Journal of Biological Chemistry, vol. 279, no. 24, pp. 25093-25100, 2004.

[29] M. T. Jack, R. A. Woo, A. Hirao, A. Cheung, T. W. Mak, and P. W. K. Lee, "Chk2 is dispensable for p53-mediated G1 arrest but is required for a latent p53-mediated apoptotic response," Proceedings of the National Academy of Sciences of the United States of America, vol. 99, no. 15, pp. 9825-9829, 2002.

[30] M. Y. Chin and G. Li, "Tissue-specific regulation of checkpoint kinase 2 expression by p53," Experimental and Molecular Pathology, vol. 75, no. 2, pp. 131-136, 2003.

[31] D. Michod and C. Widmann, "DNA-damage sensitizers: potential new therapeutical tools to improve chemotherapy," Critical Reviews in Oncology/Hematology, vol. 63, no. 2, pp. 160-171, 2007.

[32] B.-B. S. Zhou and J. Bartek, "Targeting the checkpoint kinases: chemosensitization versus chemoprotection," Nature Reviews Cancer, vol. 4, no. 3, pp. 216-225, 2004.

[33] S. D. Zabludoff, C. Deng, M. R. Grondine et al., "AZD7762, a novel checkpoint kinase inhibitor, drives checkpoint abrogation and potentiates DNA-targeted therapies," Molecular Cancer Therapeutics, vol. 7, no. 9, pp. 2955-2966, 2008. 


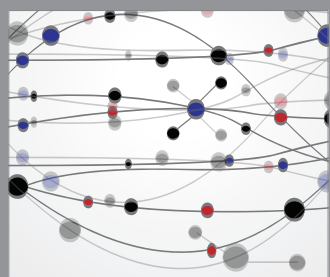

The Scientific World Journal
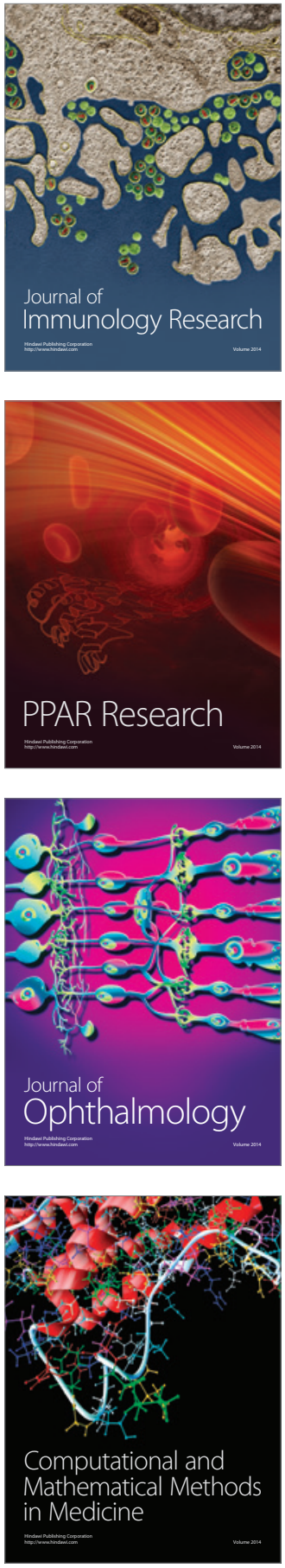

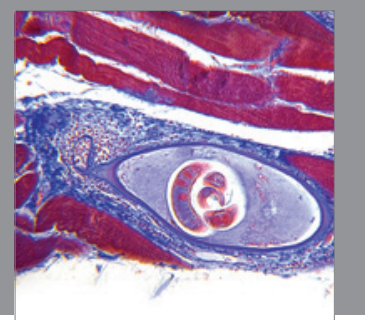

Gastroenterology

Research and Practice
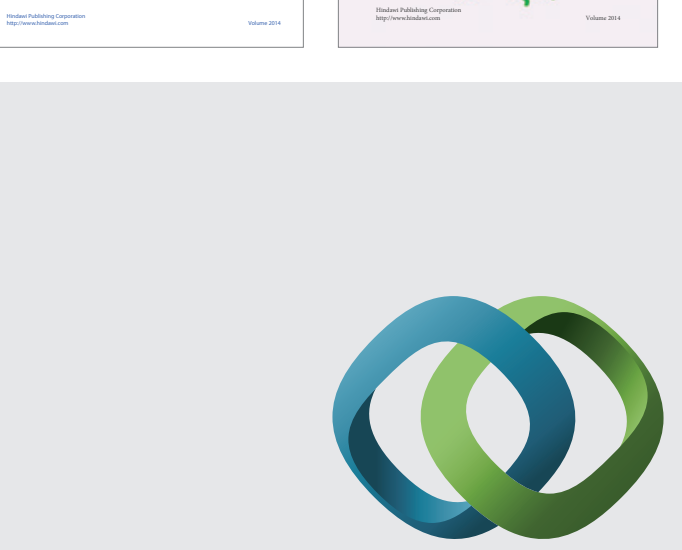

\section{Hindawi}

Submit your manuscripts at

http://www.hindawi.com
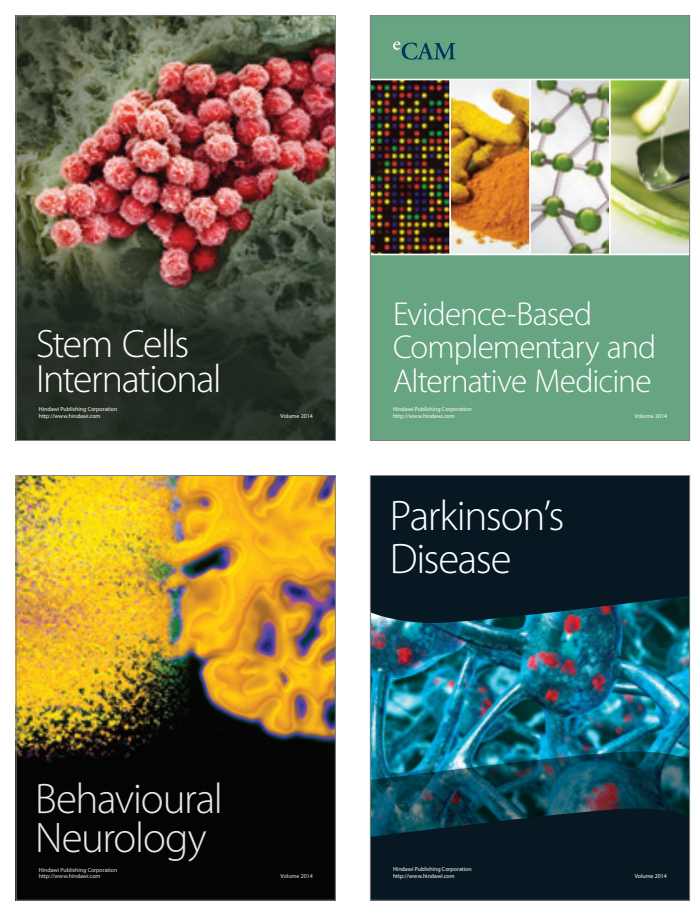

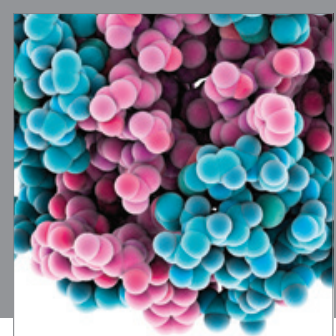

Journal of
Diabetes Research

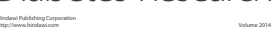

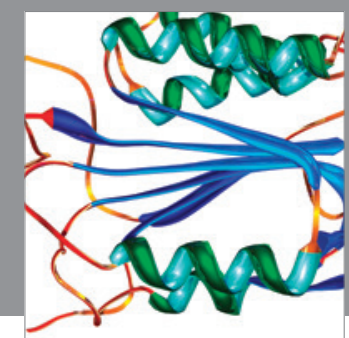

Disease Markers
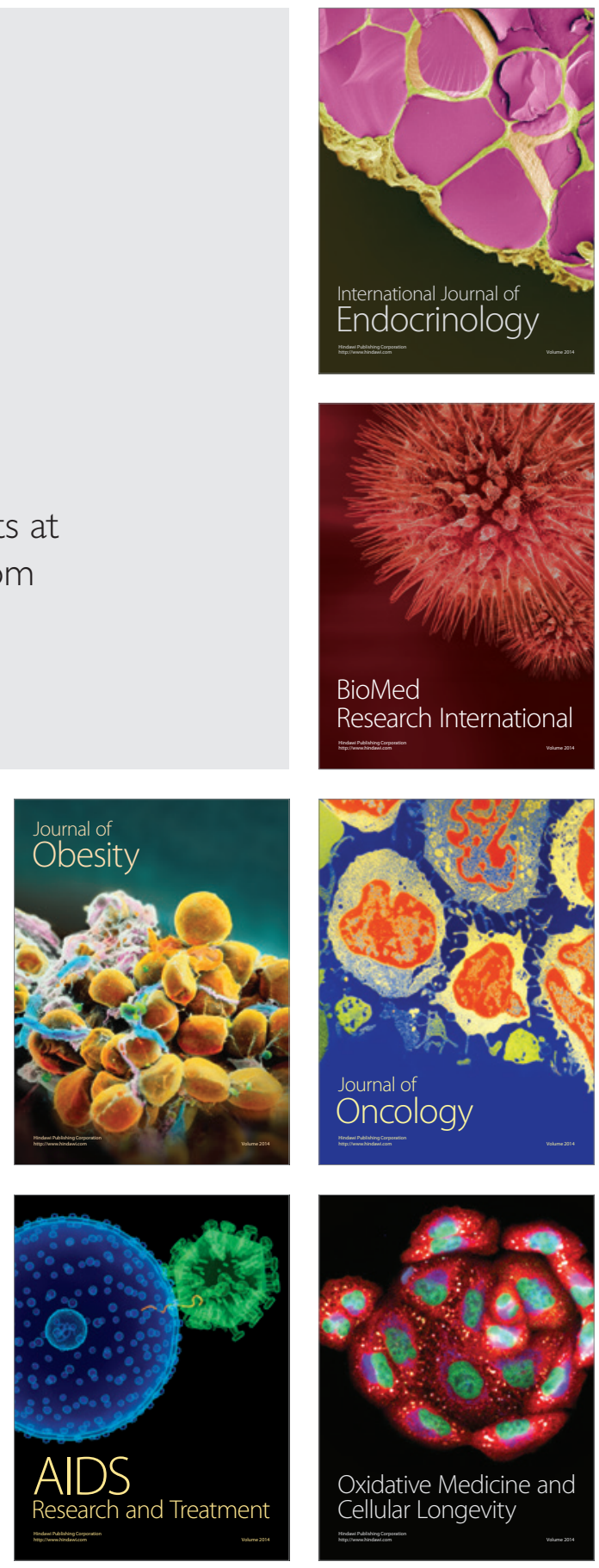